\title{
Field Study on Requirements Engineering Artefacts and Patterns
}

\author{
Daniel Méndez Fernández, Stefan Wagner, Klaus Lochmann \\ Institut für Informatik \\ Technische Universität München \\ Boltzmannstr. 3, 85748 Garching, Germany \\ mendezfe, wagnerst, lochmann@in.tum.de
}

\author{
Andrea Baumann ${ }^{1}$ \\ Fakultät für Elektrotechnik und Technische Informatik \\ Universität der Bundeswehr München \\ Werner-Heisenberg-Weg 39, 85577 Munich, Germany \\ andrea.baumann@unibw.de
}

\begin{abstract}
Requirements Engineering (RE) constitutes a critical discipline within Software Engineering. The quality of requirements is the backbone of project execution since the following phases strongly rely on it. Nowadays, industries are more then ever facing the problem that the RE process is highly volatile because it depends on the customer's capabilities, on the used process models, and on the type of specifications developed. This paper describes a study on the RE process in a specific company in the application domain of business information systems. While existing surveys often analyse the general impact of RE processes on project success, we investigate and discuss different influences that arose in 12 real-life projects and the effects of these influences onto produced RE artefacts. We infer different artefact patterns and probable project execution strategies that cause these patterns. The strategies are performed in order to tackle the different project influences. The identification enables us to get a more detailed understanding of RE in practice for the future elaboration of tailoring approaches that customise RE efforts to volatile project environments.
\end{abstract}

\section{Requirements Engineering, Strategies, Artefacts, Business Information Systems, Field Study}

\section{INTRODUCTION}

Requirements Engineering (RE) lays the foundation for successful development projects regarding cost and quality (Broy 2006). In literature as well as in practice a rich set of methods for RE is available. However, in practice these methods are still not integrated into the development process (Boehm 2006).

Even if a company defines and integrates an RE process for a broad use in different projects, it still does not address the various influences that engineers have to face in volatile project environments. The possibilities and necessities of applying available RE techniques are often limited by different project parameters such as time, budget or the availability of end users. These parameters hamper a standardised and efficient RE phase that fits individual project needs. In fact, the dependence on customer's capabilities and used process models render the process highly variable and increase the demand for a systematic customisation of $\mathrm{RE}$ efforts according to individual project environments.

Problem Statement. The high variability of the development processes makes it unclear for the practitioner what methods to choose and consequently how to design an appropriate RE process. Indeed, the

${ }^{1}$ Was at Capgemini sd\&m AG at the time of the study. understanding of what project parameters take effect on the project execution and how these effects should be reflected by the RE process is fundamental for customising the process in terms of specific strategies. Although this problem is recognised, there still exists little guidance on how to tackle it.

Research Objective. The overall research objective is to investigate how RE is performed within successful projects in order to discover strategies that allow the customisation of RE efforts to individual project environments. Since the variability in the process definition complicates a comparison and categorisation of project execution strategies, the investigation has to be performed in a process-agnostic manner.

Hence, we want to understand what problems arise in individual project environments, what consequences these problems have on produced specification documents (artefacts) and finally how volatile project situations can be reflected by artefact patterns and project execution strategies.

Contribution. Based on the analysis of real-life projects at the company Capgemini sd\&m AG we contribute

- the degree of completeness in which requirements artefacts are produced. 
- project parameters influencing the artefact completeness.

- artefact patterns reflecting probable execution strategies for RE.

A major part of the contribution is the analysis of the interrelations between these three parts. The discussion which RE execution pattern should be chosen when distinctive project parameters are given supports practical project decisions.

Context. This study is performed as part of the research cooperation between the Technische Universität München (TUM) and the research department of Capgemini sd\&m AG, a major German software and consultancy company. We perform the survey for understanding problems and necessities of RE in practice. To reach this aim, we conduct content analyses of $\mathrm{RE}$ artefacts produced in 12 real-life projects as well interviews with project participants.

\section{RELATED WORK}

Related studies can be categorised into two major areas. They either analyse the processes in order to get detailed insights into activities, used techniques and the relation to projects' success in general or they focus on assessment and improvement of RE processes.

Process Understanding. Related work analysing the RE process for a better understanding and extraction of best practices often emphasises used techniques in practice (Boehm and Alexander 1998) and the impact of used techniques on the projects' failures in general. A widely known empirical evaluation of such project failures and their causes is presented in the chaos report of the Standish Group International (1995). The chaos report examines project failures and related causes like missing user involvement. However, the report does not give detailed insights into the study design. Instead, the success study of Buschermöhle et al. (2006) gives a similar investigation of German companies including a description of how the study exactly has been performed. Still, both surveys exclusively investigate failed projects and general causes, thus, give not necessarily an understanding on how specific problems are tackled in successful projects.

Nikula et al. (2000) present a survey on requirements engineering at organisational levels of small and medium size companies in Finland. They concluded that even basic knowledge about RE is often missing in such companies and that more effort on technology transfer should be spent. This study was restricted to interviews and thereby did not give fine-grained views onto the documentation of single projects. Such a view as part of project success stories is given in Kamata and Tamai (2007) presenting an analysis of used documentation parts of the IEEE software requirements specification Std. 830-1998 in general relation to project success.
An investigation of project-specific influences was not in scope of their work.

Process Assessment. Prominent approaches that emphasise capability aspects of RE processes are, for example, CMMI and those that are specifically elaborated for RE issues. For instance, Beecham et al. (2003) performed a survey on twelve companies and identified relations between the process capability and experienced problems within those companies. They developed - based on those results - a capability maturity model specifically for requirements engineering. Similar work is presented by Sommerville and Ransom (2005). They performed a survey on requirements engineering process capabilities and their impact on the business success in general.

Summarised, earlier studies show that basic knowledge about RE is often missing at organisational levels of companies (Nikula et al. 2000). A consequence is that projects have no reference approach for a systematic process in projects. Hence, the process capability often suffers in projects (Beecham et al. 2003) and can be directly put in relation to project failures (Standish Group International 1995).

However, while the capability of RE processes strongly depends on the maturity of the underlying work products, the investigation of the overall processes and their failure rate alone gives no detailed understanding on how specific problems are tackled in practice. In order to get such an insight into RE, it has to be analysed what has been produced and why it has been produced instead of focusing on how it was produced.

\section{FIELD STUDY DESIGN}

We organise the study according to Runeson and Höst (2009). We formulate the research questions, describe the case and subject selection as well as the data collection procedures. We then define the analysis procedure before we conclude with a description of how we ensure the validity.

\subsection{Research Questions}

The study aims at identifying three main factors in RE processes and their relationships. Figure 1 shows this triangle of the factors artefact completeness, project parameters, and pattern \& strategies. The project parameters are conditions from inside or outside the project that influence its execution. They have an influence on which artefacts are created in which completeness. The complete set of artefacts and their completeness shows which pattern or execution strategy was used in the RE process. Therefore, it can also be analysed which project parameters lead to which execution strategies. To establish this triangle, we formulate 3 research questions.

RQ 1 Which artefacts exist in which completeness? 


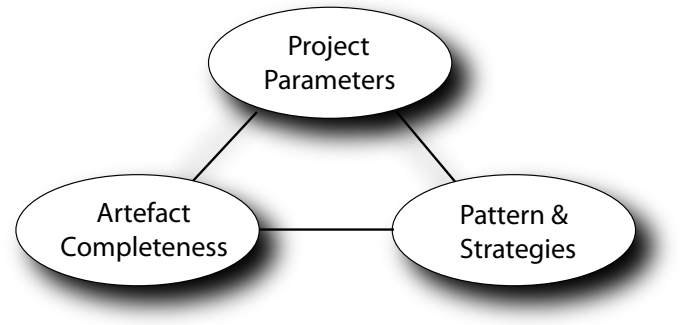

Figure 1: The triangle of factors and relationships analysed

We focus on the produced artefacts in the requirements engineering process and investigate the completeness of each artefact. It is not sufficient to just create a document and add some text, but appropriate content is needed.

RQ 2 Which project parameters have an influence on the individual process and the artefact completeness?

For a better understanding of the context of the process, influencing project parameters need to be found and put into relation to the completeness of the RE artefacts. Having the completeness of the artefacts in individual projects as well as influencing project parameters, they are set into relation. The project parameters influence the completeness of the RE artefacts and, hence, the individual project execution since the artefacts are a direct outcome of performed processes. While $R Q 1$ addresses what has been produced, RQ 2 addresses why this has been produced.

RQ 3 Are there artefact patterns and corresponding execution strategies?

Having the project parameters that influence individual projects in relation to the completeness of the $\mathrm{RE}$ artefacts, we analyse whether specific patterns can be distilled. This can be examined by comparing and categorising the overall artefact completeness of single projects in dependence to the project parameters. As a whole, this concluding analysis enables a better understanding in which contexts which RE strategy is followed in practice.

\subsection{Case and Subjects Selection}

We analyse documented and ongoing projects ${ }^{2}$ of Capgemini sd\&m AG, the German technology service entity of the Capgemini Group. The company has its focus on custom software development within the application domain of business information system. Therefore, the following study subjects and objects all come from corresponding project environments.

Regarding the study subjects (the participants), we focus on two roles: project leaders and chief analysts. Further roles like customers, could not be taken into account for reasons of confidentiality. The project leaders have the responsibility for project management issues, such

\footnotetext{
${ }^{2}$ With ongoing projects we refer to projects that have further releases or increments beyond the ones analysed.
}

as control or risk management, but also acquisition. They are the source for initial detailed information on how the development project was set up and upon what documents the project scope was defined. After the successful acquisition and scoping phase of a project, the chief analysts have the responsibility for analysing and documenting the business processes, the requirements, and the initial (overall) system specifications.

Regarding the study objects, we distinguish between the company-wide process definition (RE definition at organisational level) and the actual analysed projects that followed the process. The first serves as a preparation of the study in order to get an understanding of the used terminology and the process. The study objects include reference process descriptions, role models, training material and finally standard operating procedures. Once we understand the process definition, we select different projects and analyse corresponding documents. As a whole, 18 projects were available and ready to participate within the study, but we concentrate on 12 projects (see section 4.1 ) as these were able to offer sufficient data.

The documentation of the projects includes acquisition documents, so-called study documents, requirements specifications and system specifications. Acquisition documents capture background information and possible objectives of each project. Study documents usually capture information on the customer's domain and the organisation, the actual business processes, and a sketch about the future business processes to be supported and realised by the system under consideration. These documents mostly are produced as a preparation for a development project. In some cases such documents are produced in parallel to a maintenance phase, so that they also include a detailed description of the main use cases and technical aspects of the current IT infrastructure. The requirements specification contains the textual requirements. Finally, the system specification contains the external and internal functional specification, which defines the logical architecture of the system.

\subsection{Data Collection Procedures}

We collect the reference process definition as a preparation before analysing the single projects. The corresponding documentation was made available at the beginning of the study. We then prepare and set up the data collection from the single projects within two steps that take approximately two days for each project.

Step 1: The first step includes a short open interview of approximately one hour. This allows to get a brief overview on the project background, its relevance for the survey and an overview of the existing documentation. If the project has no relevance or if the project offers no possibility of accessing the documentation because of, e.g., non-disclosure agreements with third parties, we change the project. 
Step 2: In a second step, project participants send the documentation for a brief content analysis. This enables an initial understanding of the artefacts and allows the identification of further information needs for requesting additional documents. Within a second short open interview the preparation of the analysis is finalised and an appointment for a structured interview for the actual content analysis is made (see the next section). This way, the efforts for the study subjects within the survey can be kept to a minimum.

\subsection{Analysis Procedures}

The analysis of the projects was conducted in three steps:

Step 1 addresses RQ 1 by content analyses.

Step 2 addresses $R Q 2$ by expert interviews.

Step 3 addresses RQ 3 by cluster analysis.

Step 1: This step answers $R Q 1$ by content analyses of the collected documents, which we perform isolated without any discussions with related study subjects. This ensures an objective review of the documentation and furthermore a neutral comparison of the documentation of different projects. Within projects of a high complexity that have several thousands of requirements, we carry out single spot checks for each of the documented (software) releases. This reduces the efforts and gives an average view on the completeness of each artefact. Finally, requirement's attributes (such as the documented priority of requirements and at which basis this priority is calculated) are not explicitly taken into account.

This content analysis is performed by comparing the documents and their content on the basis of a neutral artefact-based Requirements Engineering Reference Model (REM) (Berenbach et al. 2009). It defines a taxonomy of the core RE artefacts with a description of recommended contents and an abstract description of the contents' interdependencies (traceability relations).

REM consists of three major artefacts: the Business Needs Specification, the Requirements Specification and the System Specification; each of the artefacts includes a list of content items.

On the basis of the included content items, we compare the content of each document of the projects. As REM has been developed for the application domain of embedded systems, it contains several domainspecific artefacts (e.g., hardware-specific constraints). We tailored REM according to the domain of business information systems considering also domain-specific frameworks like the Zachmann framework so that no content items where missing.

For each content item in REM completeness criteria are defined. This allows the usage of this model as a reference for a detailed analysis of a specification's content and its completeness according to the criteria:
- Completely specified: The content item can be unambiguously mapped onto a specific element of the analysed documents.

- Incompletely specified: The content item can be identified but exhibits major deficiencies to either the defined content within REM or compared to the content of other projects.

- Missing: The content item cannot be identified. The content analysis using REM gives a detailed view on the produced artefacts and their completeness (what has been documented). Still, since it allows no deeper understanding about the process and the underlying motivation (why has it been documented), we perform step 2 of the analysis.

Step 2: In order to elaborate different project parameters that have an influence on individual projects and their artefacts, a structured interview is performed. Within this interview, we ask the study subjects directly for specific requirement artefacts that we found exceptional in step 1. This allows a discussion on why specific artefacts are documented or not and furthermore the extraction of specific project parameters that are directly related to these artefacts. The interview guides through the results of the content analysis in order to (1) detect individual project parameters that took effect on the RE process and (2) detect the dependencies of those parameters to specific content items. Therefore, it addresses RQ 2 and analyses why the requirements are documented in this way. The interview is prepared by identifying the content items that exhibit differences compared to other projects and items that have not been specified at all. Based on these content items, the project-specific reasons for why they are specified in corresponding intensity are directly elaborated during a structured interview and put into relation to those content items.

Step 3: After performing the content analyses and the interviews within all the projects, we make a concluding analysis in order to address $R Q 3$. We analyse the RE artefacts of all projects for similarities in order to identify common patterns. The analysis groups the projects into clusters with similar artefact completeness. Hence, it is probable that the same project execution strategy was performed to create the artefacts of projects in one group. We thereby connect artefact patterns with project execution strategies that cause the patterns. We perform the clustering using the k-means cluster analysis, which minimises the distance from each project to the mean, called centre, in its cluster. We code the three possible verdicts for an artefact on an ordinal scale with $0=$ missing, 1 = incompletely specified, and 2 = completely specified. We experiment with different values for the number of clusters to get a useful grouping for the relation to project execution strategies. We identify suitable strategies by comparing the completeness of artefacts in the cluster and analysing the differences between them. 


\subsection{Validity Procedures}

The selection of projects from different industrial sectors, with different sizes and different project participants maximises the external validity of the results. Initial discussions with the study subjects lower barriers and ensure the collection of accurate and appropriate data. The neutral artefact model REM serves as basis for classification and the classifications are checked by other researchers. We present and discuss the results of the overall analysis with project participants. This clarifies open questions and excludes wrong results that could arise from incomplete documentation or wrong assumptions.

\section{RESULTS}

In this section the results of the study are presented. They are structured according to (1) the case and subject description and (2) according to the defined research questions.

\subsection{Case and Subject Description}

The analysed development projects can all be allocated to custom software development projects within the application domain of business information systems. Although they are restricted to this application domain, they exhibit different characteristics. Table 1 gives an overview of the analysed projects. We distinguish for each project between the industrial sectors of the customers and the project size that for reasons of confidentiality is approximated by 3 categories ${ }^{3}$. The projects are labelled with numbers and it is also described whether they are finished or are still ongoing (in terms of further releases or increments). All the projects focus either on the displacement of legacy systems, on the development of new systems or on consultancy in which application landscapes are analysed and re-designed. Consequently, all projects have in common that they define at least requirements and system specification documents independently of the following phases.

Regarding the company-wide reference process definition and the conformance of the projects to it, nearly all projects followed initially a waterfall model as a consequence of multi-staged bidding procedures. The only exception is $\mathrm{P} 4$ that followed an iterative incremental process model right from the beginning as part of a follow-up project with no explicit bidding procedure. The followed reference process definition offers a collection of architecture-driven design methods based on the Integrated Architecture Framework (IAF) of Capgemini. These methods were employed by all projects as part of

\footnotetext{
${ }^{3}$ small: 0 to 19 person years

medium: 20 to 119 person years

large: more than 120 person years
}

a so-called analysis and specification discipline according to the process framework RUP, but with no explicit assignment of an RE phase.

Table 1: Analysed projects

\begin{tabular}{lll}
\hline ID & Industrial Sector & Size $^{2}$ \\
\hline P1 (finished) & Finance & Small \\
P2 (finished) & Finance & Small \\
P3 (finished) & Finance & Small \\
P4 (ongoing) & Retail sale & Medium \\
P5 (ongoing) & Contr. authority & Medium \\
P6 (ongoing) & Telecommunication & Large \\
P7 (ongoing) & Logistics & Large \\
P8 (ongoing) & Logistics & Large \\
P9 (finished) & Aerospace & Medium \\
P10 (ongoing) & Contr. authority & Medium \\
P11 (finished) & Finance & Medium \\
P12 (ongoing) & Automotive & Large \\
\hline
\end{tabular}

\subsection{Documented Requirements Artefacts (RQ 1)}

For each analysed project, the existence and completeness of the artefacts proposed by REM is compared to the content of the analysed documents. Table 2 summarises the results of the content analysis. Going from top to bottom, the table structures the content items of REM according to the three major artefacts: the business needs, the requirements specification, and the system specification. Within the content items of the requirements specification we distinguish between functional aspects and non-functional aspects. The first includes, for example, requirements-specific application scenarios such as use case models. The non-functional models include, for example, the specification of quality requirements, models of the application's future environment, or process constraints regarding the delivery of the application ("release strategy"). Within the system specification we distinguish between the design concepts and test case specifications.

The bottom part of the table highlights traceability aspects, describing the maintained interdependencies between specific content items. We distinguish between traceability from the contents of the business needs to the requirements specification and from the requirements to the system specifications. We do not distinguish between forward tracing and backward tracing. In general, it can be observed that the content items of the initial specifications are expressed in a very different intensity. The closer we come to the system specification, the more homogeneous and detailed they are specified. In fact, the content items of the system specifications are nearly all completely specified.

Within the business needs, it can be seen that nearly all projects specified the business objectives of a customer and corresponding high-level requirements, respectively the goals (the "Customer Requirements"). However, we observe a highly variable handling of the further content 
Table 2: Completeness of artefacts in the analysed projects

\begin{tabular}{|c|c|c|c|c|c|c|c|c|c|c|c|c|c|c|}
\hline \multicolumn{2}{|c|}{ Project } & & P1 & P2 & P3 & P4 & P5 & P6 & P7 & P8 & P9 & P10 & P11 & P12 \\
\hline \multirow{10}{*}{ 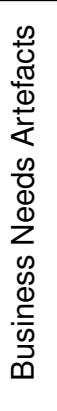 } & & Business Objectives & () & () & ( & 0 & 0 & 0 & () & () & 0 & 0 & 0 & 0 \\
\hline & & Customer/Market Requirements & 0 & 0 & 0 & 0 & 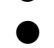 & 0 & 0 & 0 & 0 & 0 & () & 0 \\
\hline & & Value to the Customer & () & ( & ( & $\bullet$ & () & 0 & ( & 0 & () & $\bullet$ & 0 & 0 \\
\hline & & Main Features & ( & ( & ( & ( & () & $\bullet$ & ( & ( & () & ( & () & 0 \\
\hline & & Assumptions Dependencies & ( & ( & ( & ( & 0 & ( & ○ & ○ & 0 & 0 & () & 0 \\
\hline & & Scope and Limitations & 0 & 0 & 0 & 0 & 0 & ( & 0 & 0 & 0 & $\bullet$ & 0 & 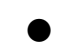 \\
\hline & & ROI Calculation & () & ( & ○ & ( & 0 & ( & 0 & 0 & 0 & $\bullet$ & ( & () \\
\hline & & Business Risk Analysis & ( & ( & ○ & 0 & 0 & 0 & 0 & $\bigcirc$ & 0 & 0 & ( & () \\
\hline & & Risk Calculation & 0 & 0 & 0 & 0 & ○ & 0 & 0 & ( & $\boldsymbol{1}$ & $\bullet$ & ( & 0 \\
\hline & & System Success Factors & 0 & $\bigcirc$ & 0 & - & 0 & ( & ( & ( & ( & ( & 0 & (1) \\
\hline \multirow{13}{*}{ 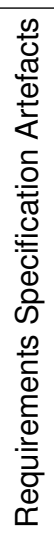 } & $\overline{\widetilde{\sigma}}$ & Application Scenarios & ( & ( & ( & () & 0 & () & ( & 0 & 0 & (1) & 0 & (1) \\
\hline & 들 & User Interface & 0 & 0 & 0 & 0 & 0 & 0 & 0 & 0 & 0 & $\bullet$ & $\bullet$ & () \\
\hline & 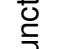 & User Classes & 0 & 0 & 0 & $\bullet$ & 0 & 0 & ( & 0 & 0 & $\bullet$ & () & $\bullet$ \\
\hline & $\stackrel{\overrightarrow{1}}{\square}$ & System Interaction & ( & ( & ( & 0 & ( & 0 & ○ & 0 & ( & ( & ( & ( \\
\hline & & Release Strategy & 0 & 0 & 0 & 0 & 0 & 0 & 0 & 0 & 0 & 0 & 0 & 0 \\
\hline & & Domain Model & - & 0 & 0 & $\bullet$ & 0 & 0 & 0 & 0 & 0 & $\bullet$ & 0 & 0 \\
\hline & $\overline{\widetilde{N}}$ & Environment Model & - & 0 & 0 & $\bullet$ & 0 & 0 & 0 & 0 & 0 & 0 & ( & 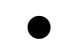 \\
\hline & $\frac{\overline{0}}{0}$ & System Boundaries & (1) & ( & 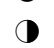 & ( & 0 & 0 & 0 & 0 & 0 & $\bullet$ & $\bullet$ & 0 \\
\hline & $\stackrel{\mathscr{c}}{5}$ & Quality Requirements & ( & - & ○ & 0 & ( & ( & ( & ( & ( & () & ( & ( \\
\hline & $\stackrel{\stackrel{L}{!}}{亡}$ & Assumptions & 0 & $\bigcirc$ & 0 & ( & ( & ( & 0 & ( & ( & 0 & 0 & $\bullet$ \\
\hline & $\bar{z}$ & SW Design Constraints & 0 & 0 & 0 & 0 & 0 & 0 & 0 & ( & 0 & 0 & () & 0 \\
\hline & & Acceptance Criteria & () & - & ○ & $\bigcirc$ & $\bullet$ & 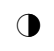 & 0 & 0 & $\bullet$ & $\bigcirc$ & (1) & $\bigcirc$ \\
\hline & & Acceptance Test Cases & 0 & $\bigcirc$ & 0 & 0 & 0 & 0 & 0 & $\bigcirc$ & 0 & 0 & 0 & $\bigcirc$ \\
\hline \multirow{14}{*}{ 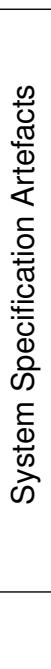 } & & Release Planning & 0 & 0 & 0 & - & 0 & 0 & 0 & 0 & 0 & 0 & 0 & 0 \\
\hline & & Behaviour Model & 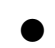 & & 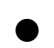 & 0 & 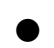 & 0 & - & 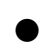 & 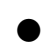 & 0 & 0 & 0 \\
\hline & & System Interaction & 0 & & 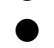 & 0 & & & & & 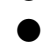 & 0 & 0 & 0 \\
\hline & O্ষ & $\begin{array}{l}\text { Service Interaction } \\
\text { Data Model }\end{array}$ & 0 & 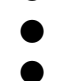 & 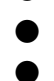 & 0 & 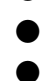 & 0 & 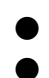 & 0 & 0 & 9 & ? & \\
\hline & U & User Interface & 0 & 0 & 0 & 0 & 0 & 0 & 0 & 0 & 0 & 0 & 0 & 0 \\
\hline & $\frac{.0}{9}$ & Communication Interfaces & 0 & 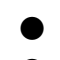 & 0 & 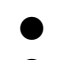 & $\bullet$ & 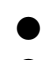 & 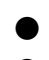 & 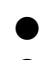 & 0 & ○ & 0 & 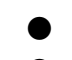 \\
\hline & ๑ & $\begin{array}{l}\text { Interfaces to Service Components } \\
\text { Architecture Constraints }\end{array}$ & 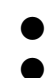 & & 0 & 0 & 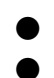 & & 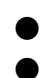 & 0 & 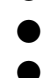 & 0 & 0 & \\
\hline & & Deployment Constraints & 0 & 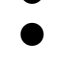 & 0 & 0 & 0 & & 0 & 0 & 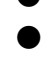 & 0 & 0 & 0 \\
\hline & & Coding Standards & - & 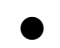 & 0 & 0 & 0 & ? & 0 & 0 & 0 & $\bullet$ & 0 & 0 \\
\hline & & Functional Test Criteria & O & 0 & 0 & O & 0 & - & (1) & 0 & 0 & 0 & (1) & 0 \\
\hline & $\stackrel{\infty}{\infty}$ & Integrations Test Criteria & 0 & & 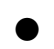 & 0 & & & (1) & (1) & 0 & 0 & () & 0 \\
\hline & & Design Constraints Test Criteria & 0 & $\bigcirc$ & 0 & 0 & 0 & ○ & ( & ( & 0 & $\bullet$ & () & $\bullet$ \\
\hline & & Traceability Needs to Requirements & O & $\bigcirc$ & O & O & O & O & O & $\mathrm{O}$ & $\bigcirc$ & 0 & 0 & 0 \\
\hline & & Traceability Requirements to Sys.Spec & 0 & $\bigcirc$ & 0 & 0 & 0 & 0 & 0 & 0 & 0 & () & ( & (1) \\
\hline
\end{tabular}


Table 3: Content items in relation to influencing project parameters

\begin{tabular}{|c|c|}
\hline Content Items & Project Parameter \\
\hline ROI Calculation & $\begin{array}{l}\text { Industrial Sector \& Confi- } \\
\text { dentiality }\end{array}$ \\
\hline Value to the Customer & Confidentiality \\
\hline Scope and Limitations & Relation to Customer \\
\hline System Success Factors & $\begin{array}{l}\text { Knowledge to Customer's } \\
\text { Domain, } \\
\text { Degree of Innovation }\end{array}$ \\
\hline Application Scenarios & $\begin{array}{l}\text { Objectives, Process Model } \\
\& \text { Availability of End Users }\end{array}$ \\
\hline Quality Requirements & $\begin{array}{l}\text { Technical Ability \& } \\
\text { Availability of Customers }\end{array}$ \\
\hline Assumptions & $\begin{array}{l}\text { Technical Knowledge of } \\
\text { Customers }\end{array}$ \\
\hline Design Concept & $\begin{array}{l}\text { Standardised Design Pro- } \\
\text { cess }\end{array}$ \\
\hline Test Criteria & External Suppliers \\
\hline
\end{tabular}

items of the business needs. Especially the "Return of Investment (ROI) calculations" and the elaboration of the customer's value is documented very variably. We observe the same for the "Scope and Limitations" that either have been completely specified or are missing. The "System Success Factors", including the basis for prioritising the requirements, are either incomplete or missing.

Within the requirements specification, we also observe a difference in the handling of the functional analysis models, especially within the "Application Scenarios". Considering the non-functional analysis models it is eyecatching that the system environment and the related content items are of high intensity. Instead, the rest of the content items, such as the quality requirements or the assumptions, are often incomplete.

Finally, the system specifications are nearly all completely specified, only the test case specifications are documented highly differently.

\subsection{Influencing Project Parameters (RQ 2)}

Within the structured interviews, we discuss the influencing parameters for specific content items that take our attention. The stated influencing project parameters vary in their project-specific details but can be summarised as done in table 3 .

Whether and how the chief analysts documented $\mathrm{ROI}$ calculations strongly depends on the industrial sector and the objectives of the customers. In particular, most customers do not share enough details of their organisation (e.g., their business processes) for performing such calculations, mostly for reasons of confidentiality. This is especially true when elaborating the value of single requirements to the customers. Consequently, the prioritisation of requirements (expressing the relevance of requirements for customers) can often not be performed. The only exception are projects that are performed with German contracting authorities. The reason is that those customers and related development projects are required to conform to the V-Modell XT, a German standard for development projects. Within this standard a ROI calculation is demanded before performing a requirements analysis.

The definition of the scope and limitations item mostly is influenced by the relation to customers. The more familiar a customer is, for example within a follow-up order, the less specific limitations are documented to improve efficiency. Similarly, the system success factors have a strong dependency on the knowledge about the customer and his domain. In particular, the less familiar customers (e.g., within the first development project for this customer), the higher the probability of defining system success factors. Dependencies are also related to the degree of innovation of the application. The higher the degree, the more specific success factors are documented.

The application scenarios within the functional analysis model exhibit dependencies to the objectives of a project in general and the process model in particular. These models are explicitely documented within a requirements specification if either a requirements phase with an acceptance phase of corresponding specifications is assigned (instead of a continuous development project) or if a change management process is set up. In the latter case the customers are often the ones to state functional requirements by the means of such analysis models, for example by the means of use cases. Another reason to be mentioned is the low availability of end users. In such cases the projects have no possibility of accessing their needs and hence the functional analysis models (e.g., the use cases) are often incomplete.

The quality requirements strongly depend on the technical ability and temporal availability of customers to state these requirements in detail. When specifying quality requirements, reference models and reference values often are missing. They remain on a high level of abstraction. For example, instead of stating specific security requirements, customers often refer to security standards, such as the Common Criteria. This project parameter has also a strong relation to the assumptions that often have to be made. Assumptions are in general used in order to compensate risks that are implied by incomplete or missing quality requirements. The less quality requirements are precisely defined, the higher the probability of defining assumptions.

Finally, the completeness of the content items of the design concept has its rationale in the standardised design process of the company according to the IAF and RUP. The completeness of the test criteria mostly depends on the integration of external suppliers, as many customers distributed the testing phase to third parties. 
Table 4: Artefact patterns and corresponding artefacts for which the completeness differ between patterns

\begin{tabular}{|c|c|c|c|c|}
\hline Pattern & 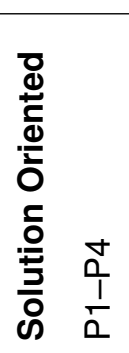 & 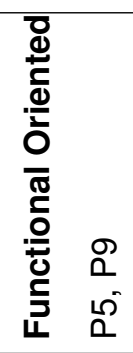 & 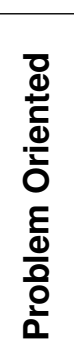 & $\begin{array}{l}N \\
\frac{1}{0} \\
1 \\
0 \\
\vdots \\
0 \\
\infty \\
0 \\
1 \\
0 \\
0\end{array}$ \\
\hline Business Objectives & (1) & $\bullet$ & & $\bullet$ \\
\hline Assumptions & ( & $\bullet$ & & ( \\
\hline Scope & - & 0 & & 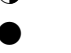 \\
\hline Business Risk & D & 0 & & () \\
\hline Risk Calculation & $\bullet$ & ( & & ( \\
\hline System Success Factors & 0 & - & & (十) \\
\hline Application Scenarios & D & - & & D \\
\hline User Interface & 0 & - & & 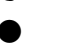 \\
\hline System Boundaries & - & $\bullet$ & & 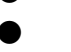 \\
\hline Assumptions & 0 & D & & D \\
\hline SW Design Constraints & $\bullet$ & $\bullet$ & & ( \\
\hline Design Const. Test Crit. & 0 & - & & 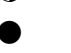 \\
\hline Acceptance Criteria & ○ & $\bullet$ & & 0 \\
\hline Acceptance Test Cases & 0 & - & & 0 \\
\hline Tracing: Needs to Req & 0 & 0 & & ( \\
\hline Tracing: Req to Sys.Spec & 0 & D & & D \\
\hline
\end{tabular}

\subsection{Artefact Patterns (RQ 3)}

To identify artefact patterns, we identify clusters with similar completeness of artefacts. The k-means cluster analysis gives the most useful grouping into three artefact patterns. The Eucledian distances from the projects to their cluster centres range from 1 to 3.3 based on the coding from 0 to 2. A higher number of clusters results in very small clusters that cannot be reasonably interpreted, but have similar distances. Table 4 shows the mapping of projects to the artefact patterns as well as the cluster centres from the k-means cluster analysis for those artefacts that have differing values between these patterns. The analysis of the three artefact patterns shows different tendencies in the artefacts. One cluster has more emphasis on the solution description, one on the functional description, and one on the problem description. This leads us to the conjecture that the artefact patterns are caused by a solution oriented, a functional oriented, and a problem oriented project execution strategy.

Solution Orientation. This pattern reflects a solutionbiased approach and implicates a weak description of the content of the business specification. The corresponding project parameter show that this pattern results from circumstances at the customer such as the relation to customers and the knowledge about their domain. The consequences are, for example, that the business objectives and the future system environment are incompletely specified and that the system success factors are missing. Instead, the projects emphasise risk calculations and the initial scope. Another consequence is that the traceability is missing due to the incompleteness of the content that would be traced.

Functional Orientation. The projects within this pattern emphasise the functional analysis models of the requirements specification including the application scenarios and the user interfaces. These circumstances are also reflected in the traceability from the requirements to the system specification. This mainly arises from the project parameter concerning the used process model, the objectives and the availability of end users. Corresponding projects have set up a change management process and end users were able to contribute to the definition of functional analysis models (like use cases). Since the functional demands requested by the customers can be linked up with the elements of the system specification this positively affects the traceability and the acceptance criteria. In contrast to the solution oriented approach, the business objectives are completely specified.

Problem Orientation. The projects in this pattern have a profound specification of the business needs, still if the requirements specification is incompletely specified. The stronger focus on business needs is also reflected in the traceability that includes in contrast to the functional oriented pattern the linkage between the requirements and the business needs. A major reason for this resulting strategy are project parameters like a high degree of (product) innovation, the relation to customers and low confidentiality issues enabling insights into customers' organisations and business processes, but also the industrial sector.

Summarised, as seen only half of the projects act problem oriented, while a third act solution oriented and a sixth functional oriented. Even if solution orientation is an established phenomenon in practice (Boehm 2006), we can, however, depict the strategy with clear artefact patterns and its clear relation to project parameters. For instance, we can observe that project parameters resulting from the customers' domain take strong influence on the choice of a solution oriented approach. The reason is that the parameters reflect the possibilities of accessing business knowledge and whether customers can contribute to a clear requirements analysis. Once, a customer does not give detailed insights into his organisation and his business processes, solution orientation can be chosen as a way to successfully tackle this problem. This circumstance is then mainly reflected by the low degree of intensity of the content items within the business needs specification. At the same time, one can observe a high rising intensity of risk calculations and of the initial scope.

Furthermore, none of the interviewees showed during the last feedback meeting awareness of having made an 
explicit decision on whether to follow solution orientation or not. This unawareness of possibilities and necessities in RE could arise from mentioned historically grown reference process models and underlying solutionbiased architecture frameworks as it is the case with the used IAF. Both still neglect the discipline of RE to be a vital part of software engineering. Hence, no integrated $\mathrm{RE}$ approach was available for its use as an orientation in the considered projects.

\subsection{Evaluation of Validity}

We subsequently discuss the construct validity, the internal validity and finally the external validity of the study.

Construct Validity. The major threat to the construct validity of our study is that we analyse the different project execution strategies only after the fact. We have not observed the study subjects in their actual execution but analysed the existing artefacts and interviewed them in retrospect. Because of that, our analysis might differ from a direct observation. However, analysing the existing artefacts gives at least a basic level of objectiveness. Furthermore, missing information was collected directly from participating study subjects to complete that picture. Another threat associated with that is that artefacts are not the only indicator for a specific project execution strategy. They do not directly tell us how the artefacts were developed. In order to mitigate this threat additional interviews and feedback meetings were held to add this additional information. Finally, for the categorisation of the artefact patterns, we had to abstract from many details of each project. Every project has its specifics because of e.g. its business contexts, or the persons that work in it that all can have a decisive influence. These detailed specifics had to be disregarded for a more general analysis. We mitigated this threat by considering these details in the interviews.

Internal Validity. In terms of the internal validity, it is possible that there were more artefacts developed in the projects that we have not analysed but which would change the classification of the project. There were, for example, specific feature lists in some projects that could not be analysed because of confidentiality. A similar threat comes from the interviews, in which the interviewees could not give all information because of confidentiality. We mitigated this in the feedback meetings in which the study subjects had the chance to comment on analysis and classification.

Furthermore, it is a threat that a large share of the information is based on the interviews and information given by people that participated in the projects. Hence, there is the possibility that the information is embellished. We mitigated this threat by the preliminary interviews in which we emphasised that the analysis is not an assessment that results in statements about which projects are good and which are bad. This lowered the barrier to giving complete and accurate information.
Finally, the analysis of the content items and their classification was done subjectively by us. This holds the threat that it is not repeatable. To mitigate that threat we employed a neutral artefact model (REM), classified the artefacts by more than one researcher, and discussed the results with study subjects.

External Validity. The results of this study can only be generalised to some extent because the major threat is that we only analysed a single company. Hence, the results can depend to a large degree on companyspecific context parameters such as the development project or the corporate culture. Moreover, all analysed projects built some kind of business information system. Hence, it is not clear to what extent the results can be transferred to other kinds of software. These threats are mitigated by analysing projects from different industrial sectors and by including the company-wide reference process definitions into the investigation. Furthermore, the analysed company is large enough that the overlap of study subjects working on more than one analysed project is low.

\section{CONCLUSIONS AND FUTURE WORK}

\subsection{Summary of Conclusions}

We analysed 12 industrial projects with regards to their requirements engineering project execution strategy. For this, we investigated the existence and completeness of $R E$ artefacts based on a generic reference model as well as used interviews for further information. This resulted in an analysis of the produced artefacts, project parameters influencing the elaboration of these artefacts and a categorisation of the projects into 3 main patterns. We found that half of the projects act problem oriented, a third act functional oriented and a sixth solution oriented.

\subsection{Relation to Existing Evidence}

In contrast to earlier studies (see section 2) we analysed projects that were successful in terms of analysing software releases being deployed to the customer and being used in production. A finding was that only $50 \%$ of these successful projects acted problem oriented in requirements engineering. First, this manifests the idea that there still is a tendency to solution orientation as it was already observed during the late 1970's by Boehm (2006). Second, we also showed that many project parameters that are considered to be the main cause for project failures (1) arise from the customer domain and often cannot be avoided, (2) can be tackled and finally (3) are reflected by specific artefact patterns. The analysis was performed in a process-agnostic manner investigating which $R E$ artefacts had been produced and what influencing project parameters took effect in the production of each single artefact. Hence, we get a more fine-grained view onto the parameters that extend existing ones. For instance, the Standish Group 
International (1995) states that $12.4 \%$ of project failures are caused by missing user involvement. We showed not only that these problems can be tackled, we also detailed this parameter by the availability of end users, their technical ability, confidentiality issues, and the general relation to the customers, each having different impacts on different artefacts. Further discovered parameters that are not in scope of current studies are, for example, the industrial sector, the degree of innovation (of the product), or the involvement of external suppliers.

\subsection{Impact/Implications}

What is a successful artefact pattern in requirements engineering depends on the parameters that influence the project. We found in the study that in successful projects these parameters, such as confidentiality or technical ability, lead to differences in artefacts and finally artefact patterns. The identified project execution strategies that are reflected by the artefact patterns can all be applied in real-life projects in order to adapt the $R E$ process to individual needs of volatile project environments.

\subsection{Limitations}

The survey has only been performed within one company. If extending this survey to other companies, this could affect the results and consequently the criteria for deriving the patterns. We doubt that the basic findings would change extremely but we would be able to make more detailed assertions on details of the patterns based on elaborated trends. Furthermore, we only analysed successful projects and, hence, cannot investigate the differences in patterns.

\subsection{Future Work}

As we concentrate on a single company, it is important that this study is extended by further projects and other companies. In addition, all projects have been a success in the sense that they resulted in systems that are now in production at the customers. Hence, the different project execution strategies were no principle success factor. Therefore, as a future work we investigate the detailed economic effects of these different strategies, especially in combination with the identified influencing project parameters.

\section{Acknowledgement}

We acknowledge the effort of all employees of Capgemini sd\&m that participated in the study. In particular, we thank Gerhard Koller for the discussions and fruitful remarks on the paper. Finally, we are grateful to Eva Geisberger for the discussions on REM's usage for the content analyses.

\section{REFERENCES}

S. Beecham, T. Hall, and A. Rainer. Software process improvement problems in twelve software companies: An empirical analysis. Empirical software engineering, 8(1):7-42, 2003.

B. Berenbach, D. Paulish, J. Kazmeier, and A. Rudorfer. Software \& Systems Requirements Engineering: In Practice. McGraw-Hill Osborne Media, 2009.

B. Boehm. A view of 20th and 21st century software engineering. In Proc. 28th International Conference on Software Engineering (ICSE 06), pages 12-29. ACM Press, 2006.

B. Boehm and E. Alexander. Software requirements negotiation: some lessons learned. In Proc. 20th International Conference on Software engineering (ICSE '98), pages 503-506. IEEE Computer Society, 1998.

M. Broy. Requirements engineering as a key to holistic software quality. In Proc. 21th International Symposium on Computer and Information Sciences (ISCIS 2006), volume 4263 of LNCS, pages 24-34. Springer-Verlag, 2006.

R. Buschermöhle, H. Eckhoff, and B. Josko. Success Erfolgs- und Misserfolgsfaktoren bei der Durchführung von Hard- und Softwareentwicklungsprojekten in Deutschland. BIS-Verlag, 2006.

Capgemini. Integrated architecture framework. www.capgemini.com/iaf.

M. Itakura Kamata and T. Tamai. How does requirements quality relate to project success or failure? In Proc. 15th IEEE Requirements Engineering Conference, pages 67-78. IEEE Computer Society, 2007.

U. Nikula, J. Sajaniemi, and H. Kälviäinen. A stateof-the-practice survey on requirements engineering in small-and medium-sized enterprises. Research Report 1, Telecom Business Research Center Lappeenranta, 2000.

P. Runeson and M. Höst. Guidelines for conducting and reporting case study research in software engineering. Empirical Software Engineering, 14(2): 131-164, 2009.

I. Sommerville and J. Ransom. An empirical study of industrial requirements engineering process assessment and improvement. ACM Transactions on Software Engineering and Methodology, 14(1):85117, 2005.

Standish Group International. The Chaos Report. http://net.educause.edu/ir/library/pdf/NCP08083B.pdf, accessed on 2009/03/15, 1995. 